\title{
A Neutron Star-White Dwarf Binary Model for Repeating Fast Radio Burst 121102
}

\author{
Wei-Min $\mathrm{Gu}^{1}$, Yi-Ze Dong ${ }^{1}$, Tong Liu ${ }^{1,2}$, Renyi $\mathrm{Ma}^{1}$, and Junfeng Wang ${ }^{1}$
}

\begin{abstract}
We propose a compact binary model for the fast radio burst (FRB) repeaters, where the system consists of a magnetic white dwarf (WD) and a neutron star (NS) with strong bipolar magnetic fields. When the WD fills its Roche lobe, mass transfer will occur from the WD to the NS through the inner Lagrange point. The accreted magnetized materials may trigger magnetic reconnection when they approach the NS surface, and therefore the electrons can be accelerated to an ultra-relativistic speed. In this scenario, the curvature radiation of the electrons moving along the NS magnetic field lines can account for the characteristic frequency and the timescale of an FRB. Owing to the conservation of angular momentum, the WD may be kicked away after a burst, and the next burst may appear when the system becomes semi-detached again through the gravitational radiation. By comparing our analyses with the observations, we show that such an intermittent Roche lobe overflow mechanism can be responsible for the observed repeating behavior of FRB 121102.
\end{abstract}

Subject headings: accretion, accretion disks — binaries: general — gravitational waves - magnetic reconnection — white dwarfs

\section{Introduction}

Fast radio bursts (FRBs) are millisecond-duration radio pulses with mysterious physical origin. With the exception of one FRB detected by the Arecibo telescope (Spitler et al. 2014) and one by the Green Bank Telescope (GBT), all the observed FRBs were detected by the Parkes telescope (e.g., Lorimer et al. 2007; Keane et al. 2012; Thornton et al. 2013; Keane et al. 2016). The observed large values of dispersion measure indicate that FRBs are probably extragalactic origin (e.g., Katz 2016). Several models have been introduced to interpret

\footnotetext{
${ }^{1}$ Department of Astronomy, Xiamen University, Xiamen, Fujian 361005, China; guwm@xmu.edu.cn

${ }^{2}$ Department of Physics and Astronomy, University of Nevada, Las Vegas, NV 89154, USA
} 
FRBs, including giant flares from a magnetar (Popov \& Postnov 2010; Kulkarni et al. 2014), giant pulses from a young pulsar or a magnetar (Cordes \& Wasserman 2016; Pen \& Connor 2015; Lyutikov et al. 2016), the merger of neutron stars (NSs) or white dwarfs (WDs) (Totani 2013; Kashiyama et al. 2013; Wang et al. 2016), the collapse of a massive NS into a black hole (BH) (Falcke \& Rezzolla 2014; Zhang 2014), the collision between an NS and an asteroid or a comet (Geng \& Huang 2015), and the collapse of charged BHs (Zhang 2016a,b; Liu et al. 2016).

Recently Spitler et al. (2016) showed ten additional bursts from FRB 121102 by Arecibo from May to June, 2015. Moreover, Scholz et al. (2016) showed six additional bursts from November to December, 2015, among which five bursts were detected by GBT and one by Arecibo. Thus, for this unique known repeating FRB, there are totally 17 observed bursts as shown in Table 2 of Scholz et al. (2016). Obviously, the repeating bursts can rule out the models involving cataclysmic events such as the merger of compact stars or the collapse of a massive NS into a BH. As mentioned in Scholz et al. (2016), the probable extragalactic distance and repeating behavior may favor giant pulses from a young pulsar or a magnetar (Cordes \& Wasserman 2016; Pen \& Connor 2015), or radio counterparts to magnetar X-ray bursts (Lyutikov 2002; Popov \& Postnov 2013; Katz 2015). In addition, Dai et al. (2016) suggested an interesting model that a repeating FRB originates from a highly magnetized pulsar encountering with lots of asteroids in an asteroid belt. Very recently, Wang \& Yu (2016) studied the frequency distributions of peak flux, fluence, duration and waiting time for this repeating source.

In this Letter, we propose a different model to understand the repeating behavior of FRB 121102. Our binary system consists of a magnetized WD and an NS with strong bipolar magnetic fields. We show that the intermittent Roche lobe overflow mechanism can be responsible for the repeating behavior. The remainder of this Letter is organized as follows. A compact binary model is illustrated in Section 2. Analyses of the time interval between two adjacent bursts are studied in Section 3. Application of such a model to the repeating FRB 121102 is presented in Section 4. Conclusions and discussion are made in Section 5.

\section{Compact binary model}

In this section we propose a compact binary model for FRB repeaters. The binary system consists of a magnetic WD and an NS with strong bipolar magnetic fields. As illustrated in Figure 1, when the WD fills its Roche lobe and the system is therefore called semi-detached, mass transfer will occur from the WD to the NS. The materials in the atmo- 
sphere (the yellow region) of the WD can pass through the inner Lagrange point ( $L_{1}$ point) and then be accreted by the NS, as shown in Figure 1(a). When the accreted magnetized materials approach the NS surface, magnetic reconnection may be triggered and therefore the electrons can be instantaneously accelerated to an ultra-relativistic speed (e.g., Zhang \& Yan 2011). Consequently, strong electromagnetic radiation can be released by the curvature radiation as the electrons move along the NS magnetic field lines. On the other hand, as studied by King (2007) based on the conservation of angular momentum, the mass transfer will be a continuous behavior for $q>2 / 3$, where $q$ is defined as the mass ratio of the WD to the NS. On the contrary, for $q<2 / 3$, the WD may be kicked away and the system may become detached after a Roche lobe overflow process, as shown in Figure 1(b). The system can become semi-detached again through the gravitational radiation and therefore the next transfer process and corresponding magnetic reconnection can restart. Thus, for the case of $q<2 / 3$, the Roche lobe overflow may be an intermittent type. Since the mass of an NS is normally larger than $1.4 M_{\odot}$ and the mass distribution for WDs is peaked at $0.6 M_{\odot}$, the intermittent type of Roche lobe overflow may be a common phenomenon for the semi-detached NS-WD systems.

We would point out that the cataclysmic variable (CV) is an analogous system. However, accretion in CVs is continuous rather than episodic. In our opinion, the main difference is that, in our model the accreted materials are from a WD instead of a main sequence star. We may therefore expect a much more violent mass transfer than in CVs. As a consequence, the outward moving due to the conservation of angular momentum may dominate over the inward moving owing to the gravitational radiation, and therefore the WD may be kicked away after a mass transfer process. We would stress that the violent mass transfer and the corresponding kick-away behavior is a fundamental assumption in our model.

The characteristic frequency of the curvature radiation of relativistic electrons is expressed as

$$
\nu_{\mathrm{c}}=\frac{3 c \gamma^{3}}{4 \pi R_{\mathrm{c}}}=1.5\left(\frac{\gamma}{60}\right)^{3}\left(\frac{R_{\mathrm{c}}}{10^{6} \mathrm{~cm}}\right)^{-1} \mathrm{GHz},
$$

where $\gamma$ is the Lorentz factor of electrons, and $R_{\mathrm{c}}$ is the curvature radius. The above equation means that, for a typical radius $R_{\mathrm{c}}=10^{6} \mathrm{~cm}$, an FRB with $\nu_{\mathrm{c}}$ of order $\sim \mathrm{GHz}$ requires $50 \lesssim \gamma \lesssim 100$, which is a reasonable range according to the acceleration by magnetic reconnection (e.g., Zhang \& Yan 2011; Kowal et al. 2012).

The duration of an FRB may be regarded as around the timescale of a magnetic reconnection. According to our model, the energy-released region is near the NS surface, and the duration $t_{\mathrm{w}}$ can be estimated by the ratio of the NS radius $R_{\mathrm{NS}}$ to the Alfvén speed 
$v_{\mathrm{A}}\left(=B_{\mathrm{NS}} / \sqrt{4 \pi \bar{\rho}}\right):$

$$
t_{\mathrm{w}}=\frac{R_{\mathrm{NS}}}{v_{\mathrm{A}}}=1.1\left(\frac{R_{\mathrm{NS}}}{10^{6} \mathrm{~cm}}\right)\left(\frac{B_{\mathrm{NS}}}{10^{11} \mathrm{G}}\right)^{-1}\left(\frac{\bar{\rho}}{10^{3} \mathrm{~g} \mathrm{~cm}^{-3}}\right)^{\frac{1}{2}} \mathrm{~ms},
$$

where $B_{\mathrm{NS}}$ is the magnetic flux density, and $\bar{\rho}$ is the averaged mass density of accreted materials. Equation (2) shows that, for typical values $R_{\mathrm{NS}}=10^{6} \mathrm{~cm}, B_{\mathrm{NS}}=10^{11} \mathrm{G}$, and $\bar{\rho}=10^{3} \mathrm{~g} \mathrm{~cm}^{-3}$, an FRB will be a millisecond duration.

Here, we would stress that the mass density in the atmosphere of a WD varies continuously from large interior values to essentially zero. In our analyses, for simplicity, we adopt an averaged mass density $\bar{\rho}=10^{3} \mathrm{~g} \mathrm{~cm}^{-3}$ for the accreted materials. In Section 5, we will discuss the degeneracy of $\bar{\rho}$ with other physical quantities in our model.

\section{Time interval analyses}

In this section we investigate the analytic variation of the time interval between two bursts with the transferred mass during the former burst. We assume a circular orbit for the analyses, i.e., the eccentricity $e=0$. The orbital angular momentum $J$ of a binary system takes the form

$$
J=M_{1} M_{2}\left(\frac{G a}{M}\right)^{\frac{1}{2}}
$$

where $M_{1}$ and $M_{2}$ are respectively the NS and WD mass, $M=M_{1}+M_{2}$ is the total mass, and $a$ is the binary separation. When the WD fills its Roche lobe, mass transfer will occur from the WD to the NS. We assume $\Delta M_{2}$ as the transferred mass during one burst, where $\Delta M_{2}$ is negative. Then, the orbital angular momentum carried by the accreted materials can be expressed as

$$
\Delta J=-\lambda \Delta M_{2} \Omega\left(b_{1}-a_{1}\right)^{2},
$$

where $\Omega$ is the orbital angular velocity, $a_{1}$ is the distance between the NS and the center of the mass, $b_{1}$ is the distance between the NS and the $L_{1}$ point, and $\lambda$ is a parameter probably in the range $0 \leqslant \lambda \leqslant 1$. The expression of $b_{1}$ takes the form (e.g., Chap. 4.4 of Frank et al. 2002)

$$
\frac{b_{1}}{a}=0.5-0.227 \log q,
$$

where $q \equiv M_{2} / M_{1}$. For $0.1 \lesssim q \lesssim 0.8$ it is often convenient to adopt a simple form to describe the Roche lobe radius of the secondary $M_{2}$ :

$$
\frac{R_{2}}{a}=0.462\left(\frac{M_{2}}{M}\right)^{\frac{1}{3}} \text {. }
$$


The radius of a WD is expressed as

$$
R_{\mathrm{WD}}=10^{9}\left(\frac{1.402 \times 10^{33} \mathrm{~g}}{M_{2}}\right)^{\frac{1}{3}} \mathrm{~cm} .
$$

In our scenario, the mass transfer will occur for $R_{\mathrm{WD}} \gtrsim R_{2}$. After a mass transfer process, the WD may be kicked away and the next transfer can appear through the gravitational radiation. For the simple case with $e=0$, the variation of $a$ due to the gravitational radiation can be written as (Peters 1964)

$$
\frac{d a}{d t}=-\frac{64}{5} \frac{G^{3} M_{1} M_{2} M}{c^{5} a^{3}} .
$$

Based on Equations (3-8), we can derive an analytic relation between the time interval $\Delta t$ between two adjacent bursts and the transferred mass $\Delta M_{2}$ during the former burst,

$$
\Delta t=\frac{5(1+q) c^{5} a^{4}}{32 q G^{3} M^{3}}\left\{\lambda[0.5(1-q)-0.227(1+q) \log q]^{2}-(1+q)\left(\frac{2}{3}-q\right)\right\} \frac{\Delta M_{2}}{M_{2}} .
$$

The above equation enables us to obtain $\Delta t$ once $\Delta M_{2}$ is given.

\section{Application to FRB 121102}

As mentioned in Section 1, for the unique known repeating FRB 121102, there are totally 17 observed bursts, as shown in Table 2 of Scholz et al. (2016). In this section, we focus on the time intervals between two adjacent bursts measured during periods of continuous observation. Based on the data from Spitler et al. (2016) and Scholz et al. (2016), we can derive totally ten such intervals among the 17 bursts, as shown by our Table 1 . It is seen that the ten intervals are from tens to hundreds of seconds. According to our scenario, an FRB is related to a magnetic reconnection event. The physical mechanism of such reconnection may be attributed to a self-organized criticality process (Wang \& Dai 2013).

Now we can compare our analyses with the observations of FRB 121102, mainly on the relation between the time interval $\Delta t$ and the transferred mass $\Delta M_{2}$. In our model we adopt typical masses for the NS and the WD, i.e., $M_{1}=1.4 M_{\odot}$ and $M_{2}=0.6 M_{\odot}$. The corresponding separation for the critical situation $R_{\mathrm{WD}}=R_{2}$ is $a=3.41 \times 10^{9} \mathrm{~cm}$. There are two extreme cases that the accreted materials do not carry the orbital angular momentum $(\lambda=0)$, and the materials carry the Keplerian angular momentum at the $L_{1}$ point $(\lambda=1)$. Previous studies on CVs indicate that $\lambda$ is around unity. We therefore adopt $\lambda=1$ in the following analyses. Then, Equation (9) can be simplified as

$$
\Delta t=-2.68 \times 10^{10} \frac{\Delta M_{2}}{M_{\odot}} \mathrm{s} .
$$


We try to show a comparison between the analyses and the observations based on two observational quantities, i.e., the fluence $F$ and the time interval $\Delta t$. A strong magnetic field with $B_{\mathrm{WD}} \approx 3 \times 10^{8} \mathrm{G}$ is required for the WD (magnetic fields have been discovered in over $100 \mathrm{WDs}$, ranging from $10^{3} \mathrm{G}$ to $10^{9} \mathrm{G}$ ). The averaged mass density of the atmosphere is adopted as $\bar{\rho}=10^{3} \mathrm{~g} \mathrm{~cm}^{-3}$. Here, we choose $z=0.1$ according to the DM measurement, and the luminosity distance is $D_{\mathrm{L}}=463.4 \mathrm{Mpc}$ for $H_{0}=69.6, \Omega_{\mathrm{M}}=0.286$, and $\Omega_{\mathrm{vac}}=0.714$. In our model, it is difficult to estimate how many electrons have been accelerated to an ultrarelativistic speed. Alternatively, we try to evaluate the released energy by the magnetic energy carried by the accreted materials. In a real case, the magnetic diffusion and the magnetorotational instability (MRI) may occur in the accretion flow and therefore change the magnetic energy. Here, for simplicity, we just assume that the carried magnetic energy

keeps unchanged during the accretion process. Thus, we can evaluate the fluence $F$ by the total magnetic energy carried by the accreted materials:

$$
F \Delta \nu D_{\mathrm{L}}^{2} \Delta \theta=-\eta \frac{B_{\mathrm{WD}}^{2}}{8 \pi} \frac{\Delta M_{2}}{\bar{\rho}},
$$

where $\Delta \nu$ is the width of radio frequency, $\Delta \theta$ is the solid angle for an FRB, and $\eta$ is the efficiency of the released energy through the curvature radiation compared with the total magnetic energy carried by the accreted materials. We adopt $\Delta \nu=1 \mathrm{GHz}$ and $\Delta \theta=0.04 \pi$ (corresponding to $1 \%$ of the whole space) for the calculations.

A comparison of analyses with observations in the $\Delta t-F$ diagram is shown in Figure 2, where $\Delta t$ is the time interval in the rest frame. The three solid lines represent the analytic relation for the efficiency $\eta=0.001,0.01$, and 0.1 , which are derived by combining Equations (10-11). The ten color filled circles denote the observational results shown in Table 1. It is seen from Figure 2 that all the bursts are well located among the three theoretical lines, which implies that the analyses are roughly in agreement with the observations. Thus, the NS-WD binary model is likely to be responsible for the FRB repeaters.

\section{Conclusions and discussion}

In this Letter we have proposed an NS-WD binary model for the FRB repeaters. The system consists of a magnetic WD and an NS with strong bipolar magnetic fields. Mass transfer will occur through the $L_{1}$ point when the WD fills its Roche lobe. Consequently, the accreted magnetized materials may trigger magnetic reconnection when they approach the NS surface, and therefore the electrons can be accelerated to an ultra-relativistic speed. In this scenario, an FRB can be powered by the curvature radiation of the relativistic electrons moving along the NS magnetic field lines. Owing to the conservation of angular momentum, 
the WD may be kicked away after a burst, and the next burst may appear when the system becomes semi-detached again through the gravitational radiation. Our analyses have shown that such an intermittent Roche lobe overflow mechanism can be responsible for the repeating behavior of FRB 121102.

For the application of our model to FRB 121102, we would point out that the required values for physical quantities are degenerate, as indicated by Equations (2) and (11). For instance, if the averaged mass density is $\bar{\rho}=10 \mathrm{~g} \mathrm{~cm}^{-3}$ instead of $10^{3} \mathrm{~g} \mathrm{~cm}^{-3}$, these two equations can be again satisfied with $B_{\mathrm{NS}}=10^{10} \mathrm{G}$ and $B_{\mathrm{WD}}=3 \times 10^{7} \mathrm{G}$. In other words, our model can work for the repeating FRB with another group of parameters. Actually, as shown by Equation (11), either a smaller $\eta$ or a larger distance $D_{\mathrm{L}}$ may be equivalent to a smaller $B_{\mathrm{WD}}$. Thus, Section 4 just shows an example group of parameters which can work for FRB 121102. In addition, we would again stress that a fundamental assumption in our model is that, once the Roche lobe is filled, the mass transfer will be so violent that the WD can be kicked away after a mass transfer process.

In this work we have focused on the curvature radiation which mainly contribute to the radio emission. However, the synchrotron radiation may also be of importance for relativistic electrons moving in magnetic fields. In the case of $B_{\mathrm{NS}}=10^{11} \mathrm{G}$ for the NS, the characteristic frequency for the synchrotron radiation is $\nu_{\mathrm{s}}=3 \gamma^{3} e B_{\mathrm{NS}} / 4 \pi m_{\mathrm{e}} c \sim 1 \mathrm{MeV}$ for $\gamma \sim 60$. Since the energy of curvature (synchrotron) radiation is related to the component of kinetic energy of electrons parallel (perpendicular) to the magnetic field lines, we can regard the energy budget for these two radiation mechanisms is comparable. As a consequence, it is easy to estimate that the fluence of $1 \mathrm{Jy}$ ms with $1 \mathrm{GHz}$ width is equivalent to $\sim 0.06 \mathrm{eV}$ for the total energy with a square meter high-energy detector, which means that the probability of receiving one $\mathrm{MeV}$ photon by such a detector is less than $10^{-7}$. Thus, the possible gamma-ray emission by the synchrotron radiation can hardly be detected except that the FRB occurs in the Local Group.

As mentioned in Section 1, Dai et al. (2016) introduced a model related to asteroids. Their radiation mechanism is also the curvature radiation. The difference is that, the energy source for FRBs in their model is the kinetic energy of asteroids, whereas the source in our model is the magnetic energy of accreted materials. In our scenario, it is still possible for a part of the kinetic energy of accreted materials to be transferred to the electrons through the strong magnetic fields, and therefore have contribution to the radio emission. In such case, the required magnetic fields of the WD may be significantly weaker than the present assumption.

We thank Zhongxiang Wang, Bing Zhang, and Zi-Gao Dai for beneficial discussions, 
and the referee for constructive suggestions that improved this Letter. This work was supported by the National Basic Research Program of China (973 Program) under grant 2014CB845800, the National Natural Science Foundation of China under grants 11573023, 11522323, 11473022, 11473021, 11333004, 11222328, U1531130, and U1331101, and the Fun-

damental Research Funds for the Central Universities under grants 20720140532, 20720150024, 20720160023 , and 20720160024.

\section{REFERENCES}

Cordes, J. M., \& Wasserman, I. 2016, MNRAS, 457, 232

Dai, Z. G., Wang, J. S., Wu, X. F., \& Huang, Y. F. 2016, arXiv:1603.08207

Falcke, H., \& Rezzolla, L. 2014, A\&A, 562, A137

Frank, J., King, A., \& Raine, D. J. 2002, Accretion Power in Astrophysics, by Juhan Frank and Andrew King and Derek Raine, Cambridge, Cambridge University Press

Geng, J. J., \& Huang, Y. F. 2015, ApJ, 809, 24

Kashiyama, K., Ioka, K., \& Mészáros, P. 2013, ApJ, 776, L39

Katz, J. I. 2015, arXiv:1512.04503

Katz, J. I. 2016, ApJ, 818, 19

Keane, E. F., Johnston, S., Bhandari, S., et al. 2016, Nature, 530, 453

Keane, E. F., Stappers, B. W., Kramer, M., \& Lyne, A. G. 2012, MNRAS, 425, L71

King, A. 2007, Philosophical Transactions of the Royal Society of London Series A, 365, 1277

Kowal, G., de Gouveia Dal Pino, E. M., \& Lazarian, A. 2012, Physical Review Letters, 108, 241102

Kulkarni, S. R., Ofek, E. O., Neill, J. D., Zheng, Z., \& Juric, M. 2014, ApJ, 797, 70

Liu, T., Romero, G. E., Liu, M.-L., \& Li, A. 2016, arXiv:1602.06907

Lorimer, D. R., Bailes, M., McLaughlin, M. A., Narkevic, D. J., \& Crawford, F. 2007, Science, 318, 777 
Lyutikov, M. 2002, ApJ, 580, L65

Lyutikov, M., Burzawa, L., \& Popov, S. B. 2016, arXiv:1603.02891

Pen, U.-L., \& Connor, L. 2015, ApJ, 807, 179

Peters, P. C. 1964, Physical Review, 136, 1224

Popov, S. B., \& Postnov, K. A. 2010, Evolution of Cosmic Objects through their Physical Activity, 129

Popov, S. B., \& Postnov, K. A. 2013, arXiv:1307.4924

Scholz, P., Spitler, L. G., Hessels, J. W. T., et al. 2016, arXiv:1603.08880

Spitler, L. G., Cordes, J. M., Hessels, J. W. T., et al. 2014, ApJ, 790, 101

Spitler, L. G., Scholz, P., Hessels, J. W. T., et al. 2016, Nature, 531, 202

Thornton, D., Stappers, B., Bailes, M., et al. 2013, Science, 341, 53

Totani, T. 2013, PASJ, 65, 12

Wang, F. Y., \& Dai, Z. G. 2013, Nature Physics, 9, 465

Wang, F. Y., \& Yu, H. 2016, arXiv:1604.08676

Wang, J.-S., Yang, Y.-P., Wu, X.-F., Dai, Z.-G., \& Wang, F.-Y. 2016, arXiv:1603.02014

Zhang, B. 2014, ApJ, 780, L21

Zhang, B. 2016a, arXiv:1602.04542

Zhang, B. 2016b, ApJ, 822, L14

Zhang, B., \& Yan, H. 2011, ApJ, 726, 90 

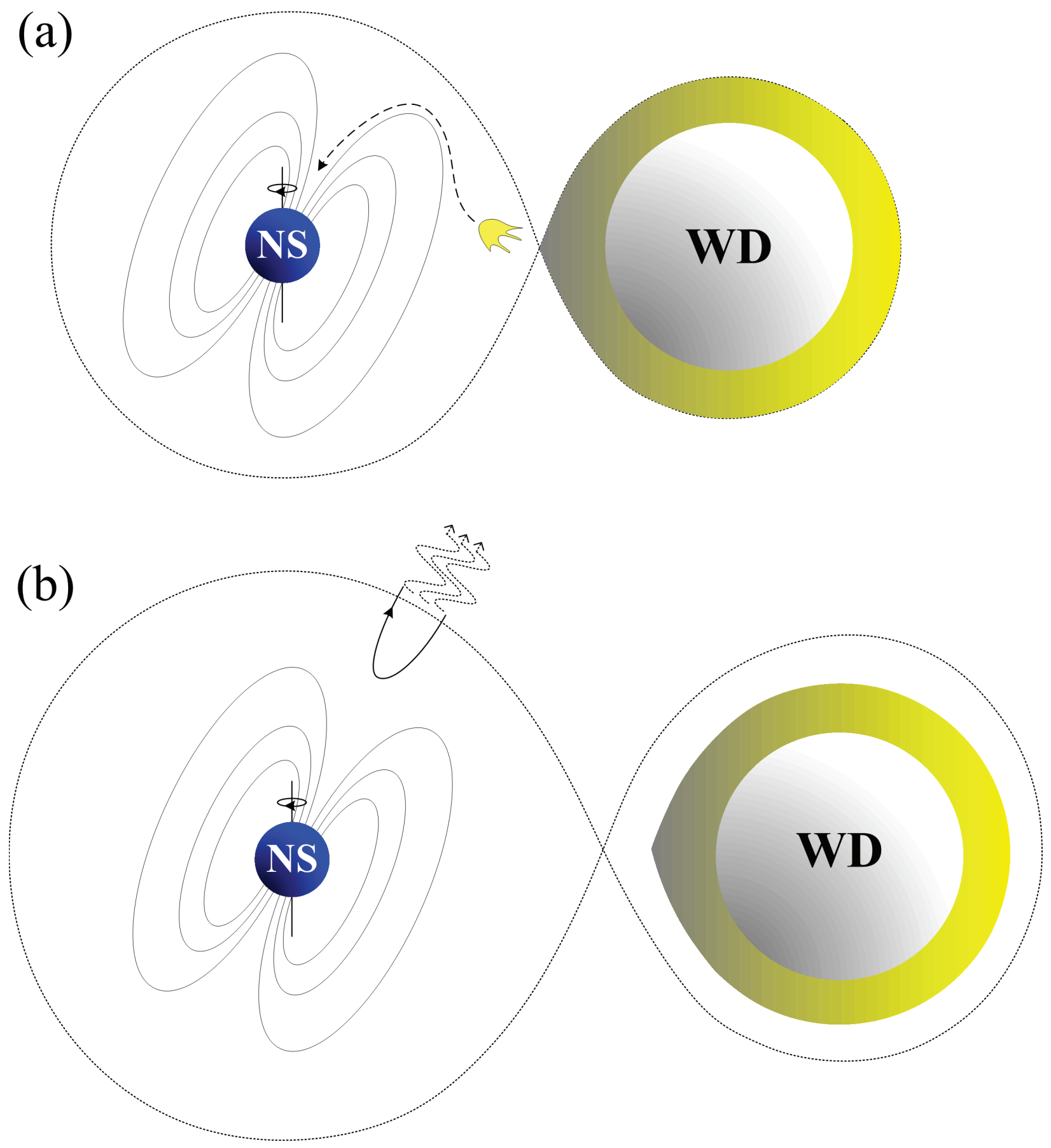

Fig. 1. - Illustration of the intermittent Roche lobe overflow in an NS-WD binary system: (a) the WD fills its Roche lobe and mass transfer occurs through the inner Lagrange point; (b) the WD is kicked away after the mass transfer, and the accreted materials trigger magnetic reconnection and strong electromagnetic radiation. 


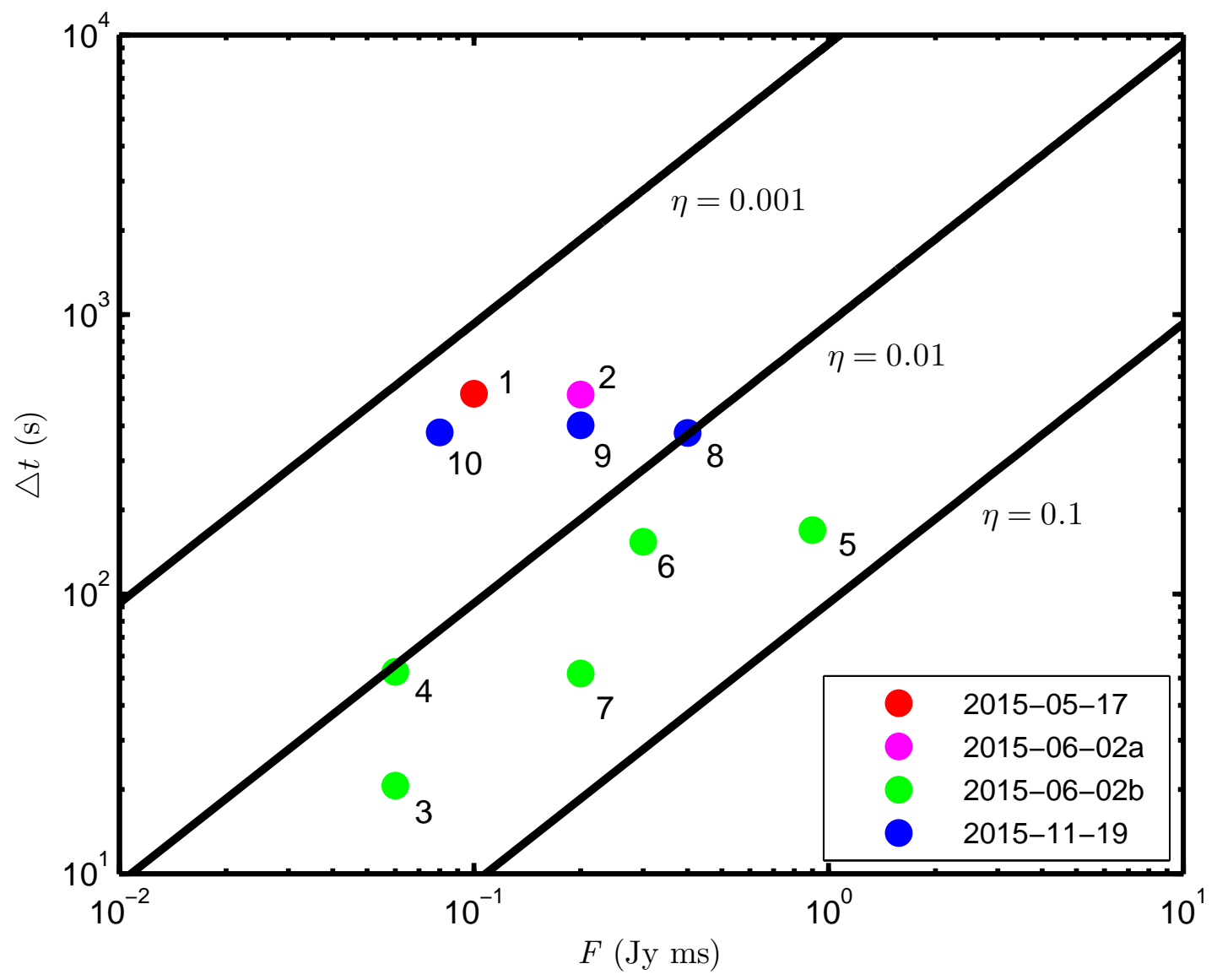

Fig. 2.- Comparison of analyses with observations in the $\Delta t-F$ diagram. The three solid lines represent the analytic relation for $\eta=0.001,0.01$, and 0.1 , and the other parameters in Equation (11) are fixed as $\Delta \nu=1 \mathrm{GHz}, D_{\mathrm{L}}=463.4 \mathrm{Mpc}(z=0.1), \Delta \theta=0.04 \pi$, $B_{\mathrm{WD}}=3 \times 10^{8} \mathrm{G}$, and $\bar{\rho}=10^{3} \mathrm{~g} \mathrm{~cm}^{-3}$. The ten color filled circles denote the observational results shown in Table 1. 
Table 1. Ten intervals in continuous observation of FRB 121102

\begin{tabular}{cccc}
\hline \hline Interval number & Date & Fluence (Jy ms) & Time interval (s) \\
\hline 1 & $2015-05-17$ & 0.1 & 572.2 \\
2 & $2015-06-02 \mathrm{a}$ & 0.2 & 568.9 \\
3 & $2015-06-02 \mathrm{~b}$ & 0.06 & 22.7 \\
4 & $2015-06-02 \mathrm{~b}$ & 0.06 & 58.0 \\
5 & $2015-06-02 \mathrm{~b}$ & 0.9 & 186.2 \\
6 & $2015-06-02 \mathrm{~b}$ & 0.3 & 169.3 \\
7 & $2015-06-02 \mathrm{~b}$ & 0.2 & 57.2 \\
8 & $2015-11-19$ & 0.4 & 414.4 \\
9 & $2015-11-19$ & 0.2 & 441.3 \\
10 & $2015-11-19$ & 0.08 & 416.3 \\
\hline
\end{tabular}

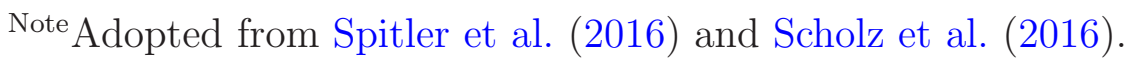

